\title{
A Prognostic Tool to Predict the Development of Postpartum Endometritis and Haematometra
}

\author{
Bulavenko $0^{1}$, Ostapiuk Lesia ${ }^{1,2 *}$, Rud V1 and Rud $0^{3}$ \\ ${ }^{1}$ Department of Obstetrics and Gynaecology, Vinnytsia National Medical University \\ of the Ministry of Health of Ukraine, Ukraine \\ ${ }^{2}$ Lviv Regional Public Health Center, Lviv, Ukraine \\ ${ }^{3}$ Country Clinical Operations, Roche Products Limited, Welwyn Garden City, United \\ Kingdom
}

*Corresponding Author: Ostapiuk Lesia, Vinnytsia National Medical University of the Ministry of Health of Ukraine, Lviv Regional Public Health Center, Lviv, Ukraine.

\author{
Received: September 03, 2020 \\ Published: September 28, 2020 \\ (C) All rights are reserved by Ostapiuk Lesia., \\ et al.
}

\begin{abstract}
Introduction: Obstetric sepsis remains one of the top 5 causes of maternal death in Ukraine, according to the data collected between 2009 - 2015. Postpartum purulent-inflammatory diseases are the reason of sepsis occurrence. Currently, there is a lack of methods of early diagnostics of postpartum endometritis (PPE).

Objectives: The main aim is to evaluate the risks of postpartum endometritis (PPE) among postpartum women and development of means of its early diagnosis using fluorescent spectroscopy. In addition, the reliability of such approach is tested via creation of a prognostic model.

Methods: Fluorescent spectroscopy was used, along with the standard diagnostic tools in order to diagnose 170 postpartum patients of the participants cohort and 40 postpartum patients of the control group with uncomplicated course of postpartum period.

Results: By statistical analysis using logistic regression, the data was evaluated according to more than 40 indicators and as a result, a prognostic model was derived. This prognostic model enables to assess the probability of development of postpartum endometritis among postpartum patients. ROC-analysis was implied in order to evaluate the quality of the proposed model.

Conclusion: Proposed multi-structured model for prognosis of the development of PPE among postpartum women, which enables determination of the patient groups susceptible to develop PPE. The major risk factors in the finalised multi-structured model of the development of PPE are: extragenital pathology, risk of miscarriage, TORCH-infections, colpitis, labour duration > 12 hrs, abnormal labour, lambda $(\lambda)$ max, fluorescence intensity $\leq 0,845$, a number of bed days and the presence of foetal distress.

It was confirmed, that the results obtained by using fluorescent spectroscopy have the highest significance among the prognostic tools used in the investigation. Therefore, application of this prognostic tool in clinical practice is a highly feasible way to improve diagnosis and prognosis of the development of postpartum endometritis. .
\end{abstract}

Keywords: Postpartum Endometritis; Fluorescent Spectroscopy; Prognostic Model; Haematometra; ROC-Analysis

\section{Abbreviations}

PPE: Postpartum Endometritis; FS: Fluorescent Spectroscopy; ROC-analysis: Receive Operating Characteristics; BS: Blood Serum

\section{Introduction}

Currently, purulent-inflammatory diseases are one of the major causes of maternal death. According to the data published by the Center of Medical Statistics MoH of Ukraine, there were around 60 cases of maternal death in 2015 (the data does not include 
the temporarily occupied territory of the Autonomous Republic of Crimea, Sevastopol and the part of the Ukrainian territory remaining under terms of the Joint Forces Operation). According to the data published by the United Nations Organisation, the index of maternal death in Ukraine is one of the highest in Europe, 24 deaths per 100,000 people; in comparison, the numbers of deaths across European countries are as follows: Poland (3), Czech Republic (4), United Kingdom (9), France (8) and Spain (5). Although being among the highest indices in Europe, Ukraine is far from the average index of maternal death in the world, being 216 deaths per 100,000 people. It was stated by the international organisations such as UNICEF, WHO, International Bank and United Nations Populations Fund that from 1990 to 2015, the level of maternal death in Ukraine has decreased by $47.8 \%$ [1].

In 2015, the leading cause of the maternal death across the world, as well as in Ukraine, was extragenital pathology. Conditions such as haemorrhage, preeclampsia, pathological delivery, sepsis were also considered as major causes of maternal death in Ukraine between 2009 and 2015, with sepsis remaining in the majority [2]. Throughout 2009 - 2012 and in 2014, sepsis was a third cause of maternal death and in 2015 it has caused $8.2 \%$ of the total maternal deaths. Extragenital pathology, preeclampsia and amniotic fluid embolism remained at top 4 maternal death causes respectively. Due to a major impact of sepsis on maternal death rate in Ukraine, we decided to investigate methods of early diagnosis of purulentinflammatory diseases, which if untreated, can consecutively lead to sepsis and death [2-5].

We carried out a thorough literature review in order to investigate the methods of prophylaxis in order to prevent the development of postpartum purulent-inflammatory diseases using antibiotics [6-8]. However, it was determined, that the early diagnosis of this condition was neglected, therefore statistical analysis is used in order to prove the effectiveness of diagnostic methods and their application in clinical practice.

\section{Aim of the Study}

The aim of this research is the assessment of the risks of emergence of postpartum endometritis (PPE) among postpartum women and implication of early diagnosis using fluorescent spectroscopy and confirmation of our hypothesis through a prognostic model.

We determined that in order to reduce the occurrence and development of PPE, the following issues have to be resolved.
Firstly, there is a need to develop clear guidelines in order to improve the techniques involved in the diagnosis of postpartum endometritis and associated diseases, primarily at their initial stages. Therefore, we decided to imply FS as a cornerstone of our strategy.

Secondly, we aimed to collect a vast amount of clinical, anamnestic and laboratory data generated during patient treatment. In order to prove the effectiveness and credibility of the achieved results, statistical methods of data analysis and forecasting were used to estimate development of purulent-inflammatory diseases.

Currently, the method of logistic regression is one of the prevalent techniques implied in prognostic model development by the scientists across the globe. However, it is essential to properly justify the implementation of this method in the medical practice and reduce the chances of having any complications related to it.

\section{Methodology}

The clinical research center for this particular investigation was the Department of Gynecology №2 of Vinnytsia Council Clinical Hospital №2. The luminescent laboratory of the Department of Experimental Physics, Ivan Franko Lviv National University was an experimental research centre. The overall research was carried out from 2015 to 2019. The research methods used in diagnosis of postpartum endometritis included clinical, laboratory, biochemical, instrumental and statistical, as well as the use of fluorescent spectroscopy (Patent for the model UA 76953 from 25.01.2013 and Patent for the model UA133472 from 10.04.2019) [9,10].

The investigation was carried out using monochromators MDR2 and MDR-12. Blood serum (BS) stimulation was done under wavelength of $280 \mathrm{~nm}$. The choice of this particular wavelength can be supported by the fact, that it detects albumin molecules, which undergo conformational changes during purulent-inflammatory diseases. The main focus of this study was to investigate the BS and involved the following patient cohorts: 65 patients with postpartum endometritis (PPE) and haematometra and 40 patients with uncomplicated course of the postpartum period. The research was carried out according to the Human Research Ethics protocol (fragment from protocol №11 of the Bioethics Committee of National Pirogov Memorial Medical University, Vinnytsia, from 19.11.2015).

\section{Data analysis}

In total, more than 40 parameters concerning the course of the pregnancy, labour, and postpartum period were analysed. This also 
includes the results of the investigations where the use of FS was implied.

Logistic regression was implied for the statistical analysis of the data. The main purpose of this method is to determine connection between several independent variables and one dependent variable.

In terms of medical research, logistic regression helps to derive a model, based on dependency between characteristics of a particular disease and its prognostic factors; this model is a cornerstone of the timely and correct diagnosis. Another potential use of this model is the evaluation of potential risk factors in clinical practice. Additionally, statistical data assessment was carried out using ROC-analysis (Receive Operating Characteristics), which is an alternative method to determine significance of clinical tests. ROCanalysis is the final stage of establishing the most efficient model; it enables determination of cut-off threshold as well helps in devising the most viable model of prognosis [11].

\section{Results and Discussion}

As a part of our investigation we were able to determine 17 core factors out of a total of 40, which preceded PPE (or appeared during its' early stages) and could potentially lead to development of this complication. Moreover, we established that the aforementioned factors may be directly or indirectly connected to purulent-inflammatory diseases. In our previous research, we developed prognostic models for patients with PPE and lactostasis [12] and PPE [13]. This paper presents the construction of a prognostic model for patients with postpartum endometritis and haematometra.

Surgical interventions, extragenital and gynaecological pathologies, complicated course of pregnancy and labour, TORCH-infection in anamnesis, colpitis, foetal distress, invasive procedures during labour and alterations in spectrofluorescent characteristics of blood serum (BS) were some of the core factors we investigated.

In order to accurately measure the intensity of the fluorescence of BS as a measurable factor, we decided to use a qualitative approach instead of a quantitative one. As a result, if the value of the patient's level of blood serum fluorescence was $\leq 0,845$, then it was noted as " 1 "; however, if this value was $>0,845$ then it was noted as " 0 ".
In terms of cohort formation, women were divided into 4 groups: under 18 years old (Group 0), 18 - 24 years old (Group 1), 25 - 34 years old (Group 2), and older than 35 years old (Group 3).

In this study, we performed and implemented a stepwise logistic regression (with forward selection) aimed to separate the factors, whose cumulative effect would have a significant effect on PPE.

A probability of PPE taking place (Q), depending on the selected factors was calculated using the following formula:

$Q=\frac{1}{1+\mathrm{e}^{-\mathrm{R}}} * 100 \%$

Where $e=2.72 \ldots$ - is the base of a natural logarithm $\mathrm{R}=$ is the quantity calculated according to the formula 1.2 , mentioned below:

$R=K+\beta_{1} x_{1}+\beta_{2} x_{2}+\ldots+\beta_{n} x_{n}$

$\mathrm{K}=$ Is a constant

$\mathrm{n}=$ The number of factors which are included in prognostic model

$\beta_{\mathrm{i}}=$ Coefficients that correspond to a number of calculated factors

$\mathrm{x}_{\mathrm{i}}=$ Corresponding numerical values of the factors.

Theoretically, Q can hold a value ranging from $0 \%$ (an impossible event) to $100 \%$ (a constantly occurring event). The meaning of $\beta_{i}$ coefficients are calculated by the software and are represented by the natural log of the correlation of the probabilities of corresponding variables. Increasing the value of the independent variable by a unit of measurement would increase the chances of developing complications in $\operatorname{EXP}(\beta)$ times.

The equation was evaluated according to Akaike information criterion (AIC) [14], verification using $\chi^{2}$ for the likelihood ratio test and by Nagelkerke's R ${ }^{2}$ (Pseudo R-squared) $[15,16]$.

Furthermore, ROC-analysis was used in order to determine a mathematical credibility of the model and calculate an optimal threshold of decision making (cut-off point). As a result, a ROCcurve was implied in order to demonstrate correlation between specificity and sensitivity. The area under the curve (AUC) was calculated to characterise the model's quality, where the scale varied between 0.5 (method is unacceptable) to 1 - 100\% which is an indication of the congruence in prognosis based on the model. R-Studio (Version 1.1.442) was used in order to perform statistical analysis, followed by the export and processing of the data using Microsoft 
Excel.

Analysis and calculations performed via logistic regression helped us in narrowing the list of the core factors down, from 17 to just 10, which were defined as having the biggest impact on occurrence of PPE and haematometra. These core factors are: extragenital pathology (V1), risk of miscarriage (V2), TORCH-infections (V3), colpitis (V4), labour duration > 12 hrs (V5), abnormal labour (V6), lambda ( $\lambda$ ) max (V7), fluorescence intensity $\leq 0,845$ (V8), a number of bed days (V9) and the presence of foetal distress (V10).

By substituting derived coefficient $\beta$ into the equation 1.2 we can determine the R (1), and then predict the probability of PPE and haematometra occurence in the cohort involved. In order to complete the prognosis, we need to substitute the derived $\mathrm{R}$ into the equation 1.1 .

The resulting model is correct with a probability of $99 \%$

\begin{tabular}{|l|c|c|c|c|}
\hline Factor & Coefficient $\boldsymbol{\beta}$ & $\operatorname{Exp}(\boldsymbol{\beta})$ & Annotations & $\mathbf{Z}$ \\
\hline Constant & -60.0906 & & Factor & -0.39 \\
\hline Extragenital pathology & 2.0312 & 7.62 & V1 & 1.35 \\
\hline Risk of miscarriage & 3.4242 & 30.70 & V2 & 1.70 \\
\hline TORCH & 2.7883 & 16.25 & V3 & 1.26 \\
\hline Colpitis & 4.1145 & 61.22 & V4 & 2.82 \\
\hline Labour duration $>12$ hrs & 5.6757 & 291.70 & V5 & 2.41 \\
\hline Abnormal labour & 2.3233 & 10.21 & V6 & 1.08 \\
\hline Lambda $(\lambda)$ max of blood serum & 0.1475 & 1.16 & V7 & 0.32 \\
\hline Fluorescence intensity $\leq 0,845$ & 4.8687 & 130.16 & V8 & 2.98 \\
\hline Bed days & 0.7783 & 2.18 & V9 & 1.58 \\
\hline Foetal distress & 1.8336 & 6.26 & V10 & 0.92 \\
\hline
\end{tabular}

Table 1: The results of regression coefficients related to the occurrence of the postpartum endometritis among postpartum women with PPE and haematometra $(n=65)$ using logistic regression.

$\left(\mathrm{p}<0.001, \chi^{2}=116.26, \mathrm{df}=10\right)$.

By substituting derived coefficient $\beta$ into the equation 1.2 we can determine the R (3) and hence predict the probability of PPE and haematometra occurrence in main cohort.

$$
\begin{aligned}
& \mathrm{R}=-60.0906+2.0312 * \mathrm{~V} 1+3.4242 * \mathrm{~V} 2+2.7883 * \mathrm{~V} 3+ \\
& +4.1145 * \mathrm{~V} 4+5.6757 * \mathrm{~V} 5+2.3233^{*} \mathrm{~V} 6+0.1475 * \mathrm{~V} 7+ \\
& +4.8687 * \mathrm{~V} 8+0.7783 * \mathrm{~V} 9+1.8336 * \mathrm{~V} 10
\end{aligned}
$$

Nagelkerke coefficient of determination of this model equals 0.9106 (i.e. the set of variables in this model explains almost 91\% of dispersion of the dependent variable). The area under curve $(\mathrm{AUC})=0.99$.

Optimal threshold of decision making (cut-off point) which allows a maximum balance between sensitivity and specificity for this model was 0.53 . Therefore, when the risk of PPE occurrence is
$0.53 \leq$, we can conclude, that the patient has a high risk of developing PPE. Consequently, if the $0.53 \geq$, we can confirm the absence of risk of PPE development (Figure 1 and 2).

When the threshold of decision making (cut-off point) is 0.53 , the values for the parameters are as follows: sensitivity is $96.92 \%$, specificity is $97.50 \%$, the likelihood ratio of the positive result (LR+) is 38.77, the likelihood ratio of the negative result (LR-) is 0.03 , positive prognostic value (PPV) is $98.44 \%$, negative prognostic value (NPV) is $95.12 \%$. The data used in the aforementioned prognosis using threshold of decision making are outlined in table 2 .

Table 3 and 4 demonstrate the results of spectrofluorescent characteristics of blood serum and probability of developing postpartum endometritis among the main cohort.

Point for this model was 0.53 , leading to a confirmation that 


\begin{tabular}{|l|c|c|c|}
\hline a & $\begin{array}{c}\text { Uncomplicated course of postpartum } \\
\text { period (n= 40) }\end{array}$ & $\begin{array}{c}\text { Diagnosed postpartum endometritis + } \\
\text { haematometra (n = 65) }\end{array}$ & \begin{tabular}{c} 
Total \\
\hline Calculated value $\leq 0.53$
\end{tabular} \\
\hline Calculated value $\geq 0.53$ & $1(2.5 \%)$ & $2(3.08 \%)$ & 41 \\
\hline Total & 40 & $63(96.92 \%)$ & 64 \\
\hline
\end{tabular}

Table 2: Diagnostic value of the mathematical model implied in postpartum endometritis and haematometra prognosis.

\begin{tabular}{|c|c|c|c|}
\hline No & $\mathbf{I}_{\mathbf{f}}$ & $\boldsymbol{\lambda}_{\max }$ & Probability \\
\hline 1. & 1 & 335.1 & 0.00182 \\
\hline 2. & 0.81 & 333.1 & 0.022656 \\
\hline 3. & 1.31 & 333.3 & 0.000183 \\
\hline 4. & 0.84 & 335.1 & 0.063497 \\
\hline 5. & 1.16 & 333.1 & 0.010786 \\
\hline 6. & 0.95 & 335.1 & 0.000239 \\
\hline 7. & 0.99 & 333.1 & 0.000388 \\
\hline 8. & 0.91 & 330.1 & 0.016386 \\
\hline 9. & 0.92 & 333.1 & 0.001356 \\
\hline 10. & 0.96 & 334.5 & 0.098479 \\
\hline
\end{tabular}

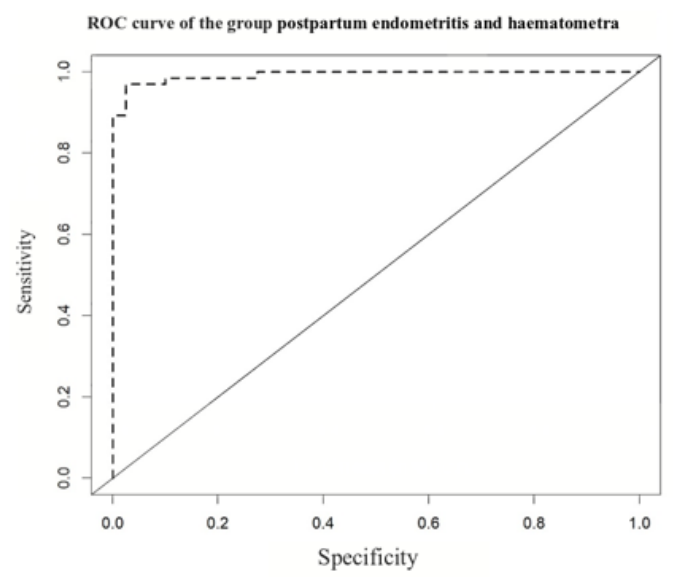

Table 3: Probability of PPE and hematometra development in control group.

\begin{tabular}{|c|c|c|c|}
\hline No & $\mathbf{I}_{f}$ & $\boldsymbol{\lambda}_{\max }$ & Probability \\
\hline 1. & 0.75 & 334.2 & 0.99999 \\
\hline 2. & 0.7 & 335.7 & 0.99985 \\
\hline 3. & 0.62 & 337.1 & 0.98931 \\
\hline 4. & 0.69 & 336.3 & 0.98798 \\
\hline 5. & 0.72 & 333 & 0.98060 \\
\hline 6. & 0.72 & 336 & 0.97998 \\
\hline 7. & 0.76 & 334.8 & 0.96802 \\
\hline 8. & 0.7 & 335.2 & 0.94889 \\
\hline 9. & 0.73 & 332.8 & 0.75735 \\
\hline 10. & 0.87 & 336.1 & 0.57308 \\
\hline
\end{tabular}

Figure 1: ROC curve for the group - postpartum endometritis and haematometra.

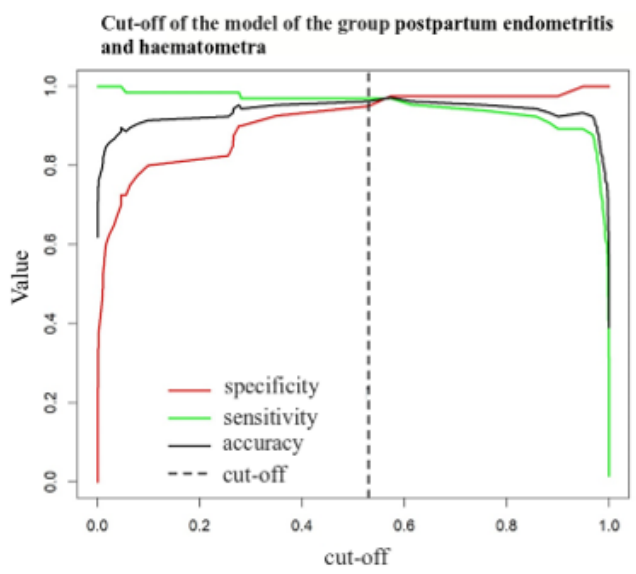

when the risk of PPE occurrence is 0.53 or higher, we can conclude that such patients are highly likely to develop PPE. As a result, if the value is less than 0.53 , we can then conclude the absence of risk of developing PPE.

Figure 2: Threshold of decision making (cut-off point) correlated to specificity, sensitivity and accuracy of the mathematical model for the group - postpartum endometritis and haematometra. 


\section{Conclusion}

1. The core factors, which have a major impact on development of postpartum endometritis and haematometra were determined as follows: extragenital pathology, risk of miscarriage, TORCH-infection, colpitis, labour duration > 12 hours, abnormal labour, lambda $(\lambda)$ max, fluorescence intensity $\leq$ 0,845 , a number of bed days and the presence of foetal distress.

2. A proposed multifactorial model which can be implied in prognosis of the risk of postpartum endometritis and haematometra occurrence also enables determination of the potential group of risk factors.

3. The probability of the risk of PPE and haematometra occurrence was defined using our mathematical model. The probability of $\geq 0.53$ enables an optimal threshold of decision making (cut-off point), which provides a balance between the specificity and sensitivity indices. Therefore, when the risk of PPE occurrence is 0.53 or higher, we can conclude, that such patients are highly likely to develop PPE. As a result, if the value is less than 0.53 , we can then conclude the absence of risk of developing PPE with $96.92 \%$ sensitivity and $97.50 \%$ specificity.

4. In order to provide access to this model of risk assessment to gynaecologists and obstetricians we came up with the software which can be used to calculate individual risks of PPE and haematometra development across individual patients.

\section{Perspective Implication of the Results}

The results of the following investigation could be used in prognosis of the risks of development of postpartum endometritis across the hospitals and relevant institutions.

\section{Bibliography}

1. Sniehirov PH. "Safety of pregnant women: a multidisciplinary approach". Ukrainian Medical Journal 4.120 7-8.

2. Smaill FM., et al. "Antibiotic prophylaxis versus no prophylaxis for preventing infection after caesarean section". The Cochrane Database of Systematic Reviews 10 (2014): CD007482.

3. Kukuruza IL., et al. "Analysis of maternal mortality from sepsis in Vinnytsia region. Ways to improve diagnosis and treatment from the standpoint of evidence-based medicine". Emergency Medicine 3 (2017).

4. Dillen Jvan., et al. "Maternal sepsis: epidemiology, etiology and outcome". Current Opinion in Infectious Diseases 23.3 (2010): 249-254.

5. Acosta CD. "Sepsis and maternal mortality". Current Opinion in Obstetrics and Gynecology 25.2 (2013): 109-116.
6. Baev OR., et al. "Antimicrobial prevention of complications after caesarean section". Questions of Gynecology, Obstetrics and Perinatology 10.4 (2011): 85-89.

7. Boichuk AV. "Mixed infection in obstetrics and gynecology and modern approaches to treatment". Emergency Medicine 6.69 (2015): 92-95.

8. Vdovychenko YuP., et al. "Rational antibiotic prophylaxis of infectious and inflammatory complications after intrauterine operations". Women's Health 3.79: 160-163.

9. Herych ID., et al. "Method of early diagnosis of purulent-septic complications using the method of fluorescence spectroscopy”. Pat. №76953 Ukraina A61V 17/00 G01N 33/48, G01N 21/64 (2013). № 201207441: 2.

10. Bulavenko OV., et al. "Method of early diagnosis of postpartum purulent-septic complications using the method of fluorescence spectroscopy”. Pat. №133472 Ukraine G01N 33/48 G01N 21/64 (2019). № 10669 (2018): 7.

11. Hryhoriev SH., et al. "The role and place of logistic regression and ROC-analysis in solving medical diagnostic problems". Journal of Infectious Diseases 8.4 (2016): 36-45.

12. Bulavenko O., et al. "Logistic Regression as a Prognostic Tool for Estimating Development of Postpartum Endometritis and Lactostasis". Acta Scientific Women's Health 1.7 (2019): 02-05.

13. Bulavenko O., et al. "Prognostic Model of Postpartum Endometritis Development”. EC Gynaecology 9.2 (2020): 01-07.

14. Shypunov AB., et al. "Visual statistics". R. M.: DMK Press (2014): 298.

15. McDonald JH. "Handbook of Biological Statistics". $3^{\text {rd }}$ edition. Sparky House Publishing, Baltimore, Maryland (2014).

16. Mangiafico SS. "An R Companion for the Handbook of Biological Statistics". Version 1.3.2 (2015).

\section{Assets from publication with us}

- Prompt Acknowledgement after receiving the article

- Thorough Double blinded peer review

- Rapid Publication

- Issue of Publication Certificate

- High visibility of your Published work

Website: www.actascientific.com/

Submit Article: www.actascientific.com/submission.php

Email us: editor@actascientific.com

Contact us: +919182824667 